\title{
Berberine-attenuated monocyte adhesion to endothelial cells induced by oxidized low-density lipoprotein via inhibition of adhesion molecule expression
}

\author{
ZHOUQING HUANG, XUELI CAI, SHENG LI, HAO ZHOU, \\ MAOPING CHU, PEIREN SHAN and WEIJIAN HUANG \\ Cardiac Center, The First Affiliated Hospital of Wenzhou Medical College, Zhejiang 325000, P.R. China
}

Received July 11, 2012; Accepted November 7, 2012

DOI: $10.3892 / \mathrm{mmr} .2012 .1236$

\begin{abstract}
Recruitment of monocytes to endothelial cells is important during early stages of atherosclerosis development. This process is predominantly mediated by cellular adhesion molecules, including vascular cell adhesion molecule-1 (VCAM-1) and intercellular adhesion molecule-1 (ICAM-1), which are expressed by activated endothelial cells in response to a number of inflammatory stimuli, including oxidized low-density lipoprotein (oxLDL). Previous studies have demonstrated that berberine, a natural extract from Rhizoma coptidis, prevents oxLDL-induced endothelial cellular apoptosis. However, its effect on the adhesion of monocytes to endothelial cells and the mechanism associated with this process remains unclear. In the present study, berberine was revealed to markedly reduce oxLDL-induced monocyte adhesion to human umbilical vein endothelial cells. In addition, the inhibitory mechanism of berberine was associated with suppression of adhesion molecule expression, including VCAM-1 and ICAM-1. Results indicate that berberine plays a protective role in the early stages of atherosclerosis.
\end{abstract}

\section{Introduction}

Recruitment of inflammatory cells to the vessel wall from the circulation and their transendothelial migration constitutes one of the earliest cellular events in the development of atherosclerotic lesions (1). Oxidized low-density lipoprotein (oxLDL), a factor known to affect vessel wall integrity (2), contributes to the development of atherosclerosis through a number of mechanisms. One mechanism is the stimulation of endothelial cell activation and the subsequent induction of expression of a

Correspondence to: Professor Weijian Huang, Department of Cardiology of Cardiac Center, The First Affiliated Hospital of Wenzhou Medical College, 2 Fuxue Road, Zhejiang 325000, P.R. China

E-mail: weijianhuang69@yahoo.com.cn

Key words: berberine, adhesion molecules, oxidized low-density lipoprotein, endothelial cells series of adhesion molecules, including intercellular adhesion molecule-1 (ICAM-1) and vascular cell adhesion molecule-1 (VCAM-1) (3-5). These adhesion molecules predominantly mediate adhesion of circulating mononuclear cells to the endothelium and facilitate their migration into the subendothelial space, thus contributing to the extravasation of mononuclear cells in the vascular wall, an initial step in the development of atherosclerosis (6,7). Moreover, cell-cell interactions between monocytes and endothelial cells further promote the development of atherosclerosis by increasing the production of specific cytokines, including tumor necrosis factor $\alpha$ (TNF- $\alpha)$ and monocyte chemotactic protein-1 (7,8). Adhesion molecules are important in the initiation and progression of atherosclerotic plaques and the suppression of secretion or expression of these adhesion molecules may be of clinical significance. Moreover, immunopharmacological inhibition of ICAM-1 and VCAM-1 has been demonstrated to inhibit the development of fatty streaks (9).

Berberine is a botanical alkaloid isolated from medicinal herbs, including Rhizoma coptidis (Huanglian) and Cortex phellodendri (Huangbai) (10). It has been extensively used in traditional Chinese medicine to treat infectious diarrhea (11). Previous studies have reported that berberine exhibits protective effects against cardiovascular diseases. For instance, berberine reduces plasma cholesterol (12) and glucose levels (13) and inhibits the expression of pro-inflammatory factors, including TNF- $\alpha$ (14). Berberine has been identified to exert antiatherogenic effects by inhibiting the expression of matrix metallopeptidase-9 (MMP-9) and extracellular matrix metalloproteinase inducer (EMMPRIN) (15). In addition, berberine has been found to inhibit generation of reactive oxygen species and prevent oxLDL-induced endothelial cellular apoptosis (16), demonstrating a protective effect on oxLDL-stimulated endothelial cells. However, the effect of berberine on the extent of monocyte adhesion to endothelial cells and the potential mechanism involved in this process remains unclear.

In the present study, the effect of berberine on the oxLDL-induced adhesion of monocytes to human umbilical vein endothelial cells (HUVECs) was investigated. In addition, the expression of adhesion molecules in oxLDL-stimulated HUVECs was analyzed to clarify the mechanism by which berberine mediates this process. 


\section{Materials and methods}

Reagents. RPMI-1640 medium, fetal bovine serum (FBS) and penicillin/streptomycin (pen/strep, 10,000 U/ml each) were obtained from Invitrogen Life Technologies (Carlsbad, CA, USA). Phorbol 12-myristate 13-acetate was purchased from Calbiochem (San Diego, CA, USA). Methyl sulfoxide (DMSO) and berberine were acquired from Sigma-Aldrich (St. Louis, MO, USA). oxLDL was purchased from Beijing Union Medical Biochemistry Room (Beijing, China). Endothelial cell medium (ECM) and human vascular endothelial growth factor (VEGF) were purchased from ScienCell (Carlsbad, CA, USA). The 3-(4,5-dimethylthiazol-2-yl)-2,5-diphenyltetrazolium bromide) (MTT) assay was obtained from Roche Diagnostics (Mannheim, Germany). TRIzol reagent for RNA isolation was purchased from Invitrogen Life Technologies. Omniscript reverse transcriptase for first-strand cDNA synthesis was obtained from Qiagen (Shanghai, China). Taq DNA polymerase was from New England Biolabs (Ipswich, MA, USA). Green fluorescent protein (GFP) adenovirus (Ad5/F35-GFP) was from Vector Gene Technology Company Ltd. (Beijing, China). VCAM-1 and ICAM-1 ELISA Kits were obtained from R\&D Systems (Minneapolis, MN, USA).

Cell culture and treatments. The human acute monocytic leukemia cell line, THP-1, was purchased from American Type Culture Collection (Rockville, MD, USA) and cultured in RPMI-1640 medium containing 10\% FBS, $10 \mathrm{mM}$ 4-(2-hydroxyethyl)-1-piperazineethanesulfonic acid (HEPES; Sigma-Aldrich) and $1 \%$ pen/strep solution at a density of $5 \times 10^{5}$ cells $/ \mathrm{ml}$ in a $5 \% \mathrm{CO}_{2}$ incubator. GFP-Ad was added to the THP-1 cells for $24 \mathrm{~h}$ to obtain GFP expression. HUVECs were purchased from ScienCell (no. 8000) and cultured in ECM containing VEGF ( $3 \mathrm{ng} / \mathrm{ml}), 10 \% \mathrm{FBS}$, $10 \mathrm{mM}$ HEPES and $1 \%$ pen/strep solution at a density of $1 \times 10^{4}$ cells $/ \mathrm{ml}$ in a $5 \% \mathrm{CO}_{2}$ incubator. The culture was then passaged 2-6 times. Growth medium was changed every other day until $80 \%$ confluence was achieved. Following this, the culture medium was replaced with ECM containing $0.4 \%$ FBS for cell starvation for $6 \mathrm{~h}$. Cells were pretreated with berberine $(5,25$ and $50 \mu \mathrm{M})$ for $2 \mathrm{~h}$, followed by oxLDL stimulation $(100 \mu \mathrm{g} / \mathrm{ml})$ for $6 \mathrm{~h}$. Cells were then cocultured with GFP-Ad-infected-THP-1 cells for $20 \mathrm{~min}$ to determine the adhesive capacity of the monocytic to endothelial cells. Unattached cells were removed and collected to calculate the percentage of cells expressing GFP.

Cell viability assay using MTT. Following the indicated treatments, cells were incubated with $0.5 \mathrm{mg} / \mathrm{ml}$ MTT in culture medium for $4 \mathrm{~h}$. The blue formazan crystals of viable cells were then dissolved in DMSO and spectrophotometrically analyzed at $570 \mathrm{~nm}$.

Flow cytometry. Flow cytometry was performed according to the manufacturer's instructions. Mean fluorescence intensity of GFP expression in THP-1 cells cocultured with HUVECs was analyzed on a BD FACSCalibur flow cytometer. The mean intensity of untreated cells was considered as $100 \%$. Changes in GFP levels in the cells following berberine treatment were evaluated and standardized against the untreated cells.

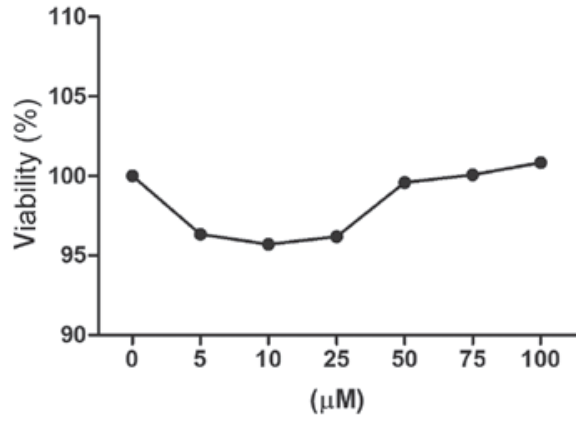

Figure 1. Effects of berberine on the viability of HUVECs. Cells were treated with various concentrations of berberine (5-100 $\mu \mathrm{M})$ and cell viability was assessed following $24 \mathrm{~h}$ using the MTT assay. Cells incubated in a medium without berberine were used as the control and considered $100 \%$ viable. HUVECs, human umbilical vein endothelial cells; MTT, 3-(4,5-dimethylthiazol-2-yl)-2,5-diphenyltetrazolium bromide.

GFP-Ad-infected THP-1 cells and endothelial cell-monocytic cell adhesion assay. Cells were cultured in 24-well plates at a density of $2 \times 10^{5}$ cells $/ \mathrm{ml}$ in a $5 \% \mathrm{CO}_{2}$ incubator and added to a $10^{6} \mathrm{GFP}-\mathrm{Ad}(2,4.5$ or $6 \mu \mathrm{l})$ titer for $24 \mathrm{~h}$ to determine the appropriate amount of GFP-Ad-infected THP-1 cell virus (multiplicity of infection, MOI). The percentage of GFP-expressing cells was then determined using flow cytometry. GFP-labeled THP-1 cells $\left(1 \times 10^{6}\right)$ were then added to each HUVEC-containing well and the incubation was continued for $20 \mathrm{~min}$. Non-adherent cells were removed using two gentle washes with phosphate-buffered saline (PBS) and the percentage of GFP mean fluorescence intensity of the cells, which indicates the number of bound THP-1 cells, was determined using flow cytometry.

$R N A$ isolation and reverse transcription-polymerase chain reaction $(R T-P C R)$. Total RNA was extracted from oxLDL-stimulated HUVECs using TRIzol reagent according to the manufacturer's instructions. Total RNA $(2 \mu \mathrm{g})$ was reverse transcribed into cDNA using a random primer and the resultant cDNA was amplified through PCR using the following primers: human VCAM-1 (575 bp), 5'-ATGCCT GGGAAGATGGTCGTGA-3' (sense) and 5'-TGGAGCTGG TAGACCCTCGCTG-3' (antisense); human ICAM-1 (370 bp), 5'-TGCCACCAATATGGGAAGGC-3' (sense) and 5'-CCG AGCTCAAGTGTCTAAAG-3' (antisense); and GAPDH, (519 bp), 5'-GGTGAAGGTCGGAGTCAACGG-3' (sense) and 5'-GTCATGAGTCCTTCCACGAT-3' (antisense). All gels were detected using the Tanon-4100 digital Gis image system (Beijing, China) and densitometric analysis was performed using Quantity One (Bio-Rad, Hercules, CA, USA) to scan the signals.

Cell enzyme-linked immunosorbent assay (ELISA). Adhesion molecule expression in HUVECs was determined by cell ELISA. HUVECs in 96-well plates were pretreated with berberine $(5,25$ and $50 \mu \mathrm{M})$ for $2 \mathrm{~h}$ and subsequently stimulated for $6 \mathrm{~h}$ with oxLDL $(100 \mu \mathrm{g} / \mathrm{ml})$. HUVECs were then washed twice with PBS and fixed with $0.025 \%$ glutaraldehyde for $10 \mathrm{~min}$. Following this, cells were incubated with $1 \mu \mathrm{g} / \mathrm{ml}$ monoclonal antibody against VCAM-1 or ICAM-1 or with $1 \mu \mathrm{g} / \mathrm{ml}$ non-specific mouse immunoglobulin G1 (IgG1; 
A

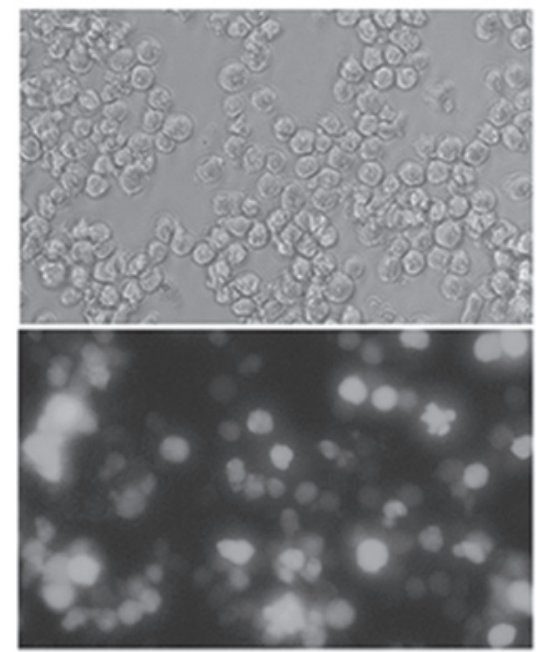

B

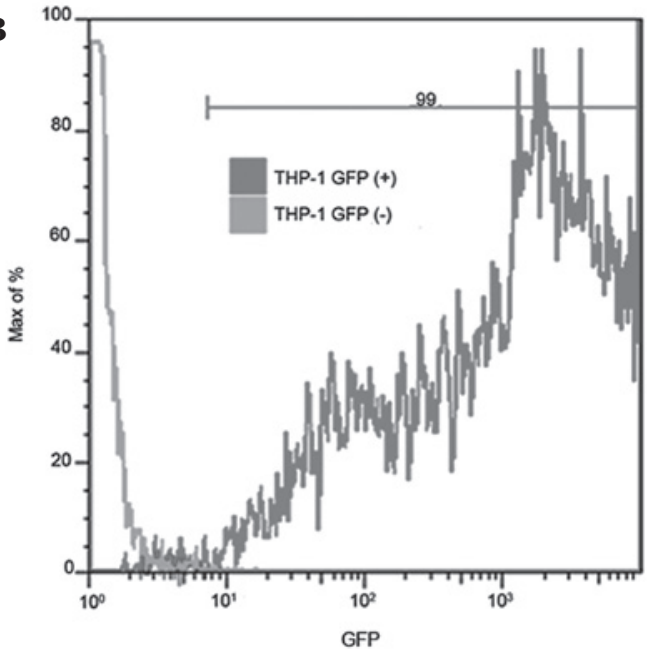

Figure 2. Infection efficiency of GFP-Ad in THP-1 cells was determined via flow cytometry. The suitable concentration of GFP-Ad-infected THP-1 cells was 5 MOI and its infection efficiency was 99\%. (A) Natural light and GFP fluorescence images in GFP-Ad-infected THP-1 cells. (B) Percentage of THP-1 cells expressing GFP fluorescence as detected by flow cytometry. GFP-Ad, green fluorescence protein adenovirus; MOI, multiplicity of infection.

Sigma-Aldrich) in PBS containing $1 \%$ bovine serum albumin (BSA) at room temperature for $1 \mathrm{~h}$. The latter was selected as the control. Following incubation, wells were washed with PBS containing $0.05 \%$ Tween-20 and incubated with horseradish peroxidase-conjugated rabbit anti-mouse IgG (1:5,000 IgG:PBS/BSA) for $1 \mathrm{~h}$ at room temperature. Cells were again washed three times with PBS:Tween and the bound antibodies were detected by incubation with $3 \% \mathrm{O}$-phenylenediamine and $0.03 \% \mathrm{H}_{2} \mathrm{O}_{2}$ at room temperature for $30 \mathrm{~min}$. Plates were read on an ELISA microplate reader at $450 \mathrm{~nm}$. The optical densities of the wells incubated with non-specific mouse IgG1 were subtracted from those of the wells incubated with monoclonal antibodies. Each experiment was performed in triplicate.

Statistical analysis. Data are presented as the mean \pm SD. Differences between treatments were determined through one-way ANOVA (LSD, S-N-K and Dunnet) using SPSS 13.0 software. $\mathrm{P}<0.05$ was considered to indicate statistically significant results. All experiments were performed at least 3 times.

\section{Results}

Effects of berberine on cell viability. The MTT assay was performed to evaluate the effect of berberine on the viability of HUVECs. Fig. 1 demonstrates that a concentration of berberine between 5 and $100 \mu \mathrm{M}$ led to no significant reduction $(\sim 0-5 \%)$ in cell viability. A berberine dose $\leq 100 \mu \mathrm{M}$ was therefore considered noncytotoxic and doses between 5 and $25 \mu \mathrm{M}$ were used in subsequent experiments.

Infection efficiency of GFP-Ad in THP-1 cells. Following incubation of THP-1 cells with GFP-Ad, the percentage of GFP expression in the cells was determined using flow cytometry. Fig. 2 shows that the suitable concentration of GFP-Ad-infected THP-1 cells was 5 MOI and its infection efficiency was $99 \%$.

Effects of berberine on the adhesive capacity of THP-1 cells to HUVECs. To investigate the adhesive capacity of THP-1 cells
A
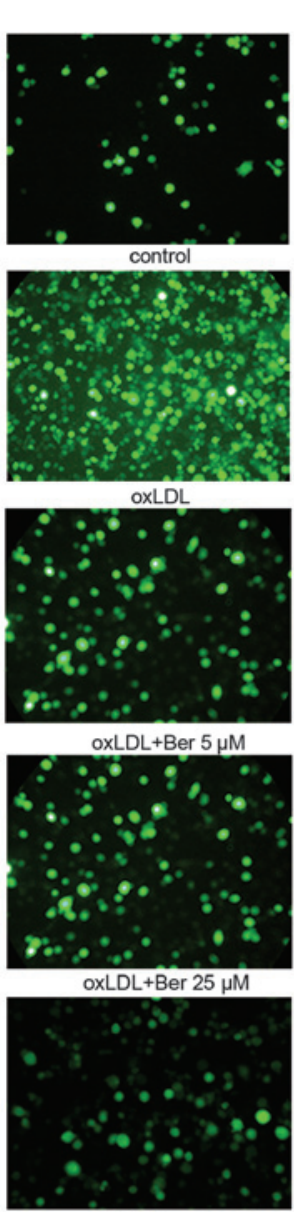

oxLDL+Ber $50 \mu \mathrm{M}$
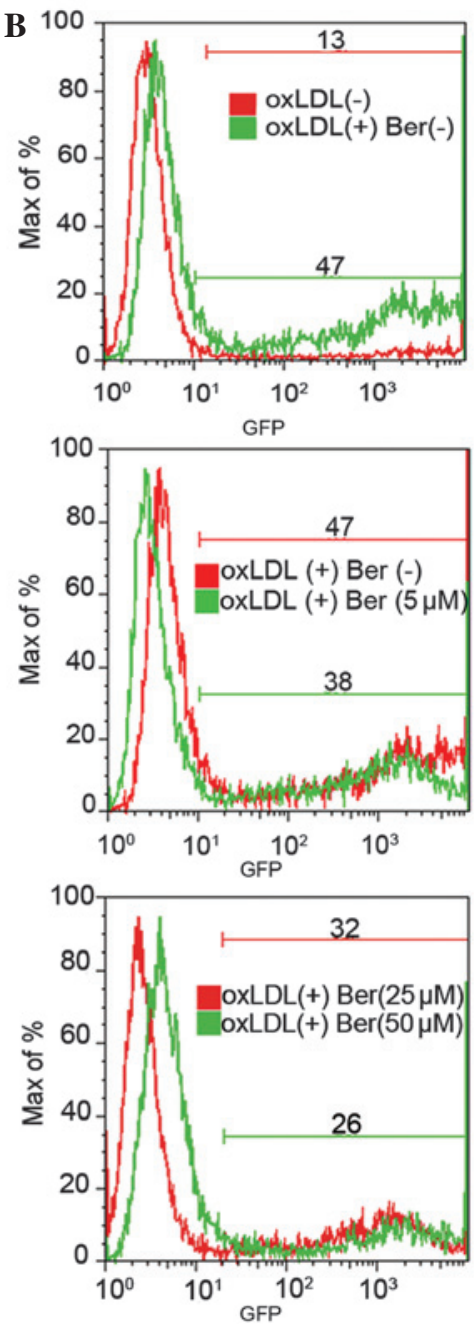

Figure 3. Effect of berberine on adhesion of THP-1 cells to HUVECs. HUVECs were pretreated with berberine for $1 \mathrm{~h}$ and stimulated with oxLDL $(100 \mu \mathrm{g} / \mathrm{ml})$ for $6 \mathrm{~h}$. The percentage of HUVEC-adhering THP-1 cells that exhibited GFP fluorescence was determined. (A) Images of cells infected with GFP-Ad. (B) Flow cytometry results of the percentage of cells expressing GFP fluorescence following berberine treatment. oxLDL, oxidized low-density lipoprotein; GFP-Ad, green fluorescence protein adenovirus; HUVECs, human umbilical vein endothelial cells. 
A

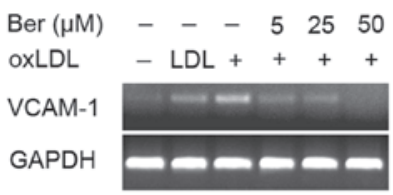

C

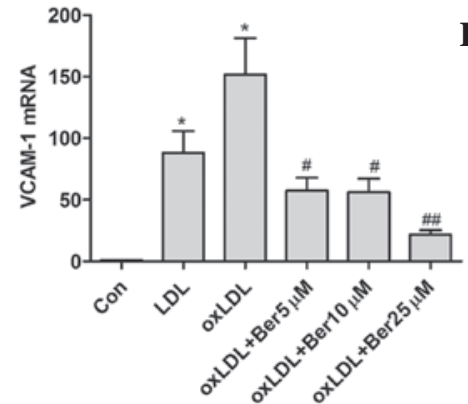

$\mathbf{E}$

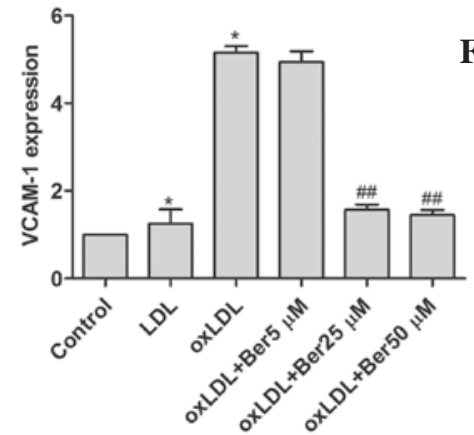

B

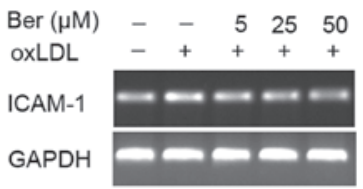

D

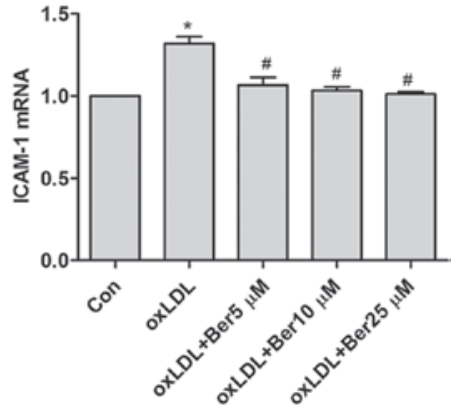

F

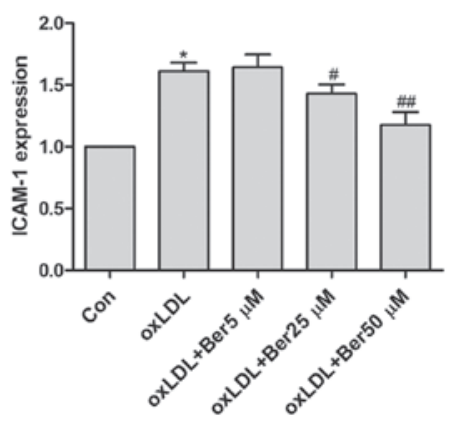

Figure 4. Effects of berberine on VCAM-1 and ICAM-1 expression in oxLDL-stimulated HUVECs. Effect of berberine on (A) VCAM-1 and (B) ICAM-1 mRNA expression. (C and D) Respective densitometric measurements. (E and F) Respective ELISA results. Data are expressed as mean \pm S.D. Band density of native HUVECs were defined as the control and set to $1 ;{ }^{*} \mathrm{P}<0.01$, vs. control; ${ }^{\#} \mathrm{P}<0.05$, ${ }^{\#} \mathrm{P}<0.01$, vs. ${ }^{\text {(*, }}$ marked group. VCAM-1, vascular cell adhesion molecule-1; ICAM-1, intercellular adhesion molecule-1; LDL, low-density lipoprotein; oxLDL, oxidized LDL; ELISA, enzyme-linked immunosorbent assay; HUVECs, human umbilical vein endothelial cells

to HUVECs, HUVECs were pretreated with berberine for $2 \mathrm{~h}$ and then stimulated with oxLDL $(100 \mu \mathrm{g} / \mathrm{ml})$ for $6 \mathrm{~h}$. The number of adherent THP-1 cells expressing GFP fluorescence was observed via flow cytometry and images were captured using fluorescence microscopy. oxLDL-stimulated HUVECs were identified to exhibit significantly increased GFP fluorescence intensity compared with the control group without oxLDL, indicating an enhanced adhesion of monocytes to HUVECs. However, berberine treatment markedly lowered the adhesive capacity of monocytes and therefore reduced the GFP fluorescence intensity of cells (Fig. 3A). Flow cytometry results (Fig. 3B) also demonstrated the same trend, wherein oxLDL treatment increased the percentage of cells that expressed GFP fluorescence compared with the control group without oxLDL treatment $(13 \pm 1.79$ vs $47 \pm 1.26 \%, \mathrm{P}<0.01)$. However, berberine pretreatment inhibited oxLDL-induced cell adhesion in a dose-dependent manner (5-50 $\mu \mathrm{M}$, from $47 \pm 1.31$ to $26 \pm 3.08 \%$, respectively).

Effects of berberine on the oxLDL-induced expression of VCAM-1 and ICAM-1 at the surface of HUVECs. OXLDL stimulated HUVECs to express VCAM-1 and ICAM-1, which led to an increase in monocyte adhesion to HUVECs. The expression of adhesion molecules was determined to elucidate the molecular mechanism of berberine inhibition on the adhesive capacity of monocytes to HUVECs. Fig. 4 demonstrates that berberine significantly suppressed VCAM-1
mRNA expression and inhibited ICAM-1 mRNA expression in the oxLDL-simulated HUVECs in a dose-dependent manner. Furthermore, ELISA results (Fig. 4E) indicate that berberine inhibited expression of VCAM-1 and ICAM-1 in oxLDL-stimulated HUVECs. The inhibition rate of VCAM-1 following treatment with 5-50 $\mu \mathrm{M}$ berberine was between -4.6 and $-22.3 \%$, respectively. ICAM-1 expression following treatment with $25-50 \mu \mathrm{M}$ berberine was between -12.3 and $-27.0 \%$, respectively. However, no significant effect was observed following treatment with $5 \mu \mathrm{M}$ berberine.

\section{Discussion}

Recruitment of monocytes to the vessel wall is an important phenomenon in the early stages of atherosclerosis (17). In the current study, berberine was observed to significantly reduce oxLDL-induced monocyte adhesion to HUVECs in a dose-dependent manner. The potential mechanisms underlying berberine suppression of the expression of adhesion molecules, namely, VCAM-1 and ICAM-1, was also clarified. Results indicate that berberine plays a protective role in the early stages of the pathogenesis of atherosclerosis.

oxLDL is a critical factor for the initiation and acceleration of atherosclerosis and its complications (18-20). OxLDL leads to endothelial dysfunction, which in turn leads to the expression of adhesion molecules $(21,22)$ and the recruitment of monocytes to the subendothelial space (20). The monocytes 
then differentiate into macrophages, resulting in transformation of macrophages into foam cells, followed by expression of a wide range of proinflammatory factors that promote the development of atherosclerosis $(17,23)$. As a positive feedback mechanism, cell-cell contact between monocytes and endothelial cells further promotes their interaction through upregulation of the expression of endothelial adhesion molecules, including VCAM-1 and ICAM-1 (8), resulting in a continual cycle of self-activation (17). Therefore, disrupting the processes that contribute to monocyte adhesion to endothelial cells is an attractive target for treatment of atherosclerosis. The present study provides direct in vitro evidence that berberine significantly attenuates VCAM-1 and ICAM-1 expression and correspondingly reduces monocyte adhesion to oxLDL-stimulated endothelial cells.

Although berberine is commonly used as an antimicrobial (11) and antitumor (24) agent in China and Korea, accumulating studies have indicated that berberine is also antiatherogenic. For example, berberine effectively improves glucose metabolism in animal models (25) and clinical studies (13). Berberine reduces plasma cholesterol (12) and inhibits expression of proinflammatory factors $(14,26)$. In addition, berberine suppresses the expression of MMP-9 and EMMPRIN in macrophages (15), disturbing the balance of collagen production and degradation and leading to plaque instability (27). These studies demonstrate that berberine exerts multiple effects on the development of atherosclerosis by reducing the major risk factors associated with atherosclerosis, including diabetes and hyperlipemia and interfering with microcellular mechanisms, i.e., expression of EMMPRIN and proinflammatory factors. The present study extends these observations by demonstrating that berberine decreases expression of the two adhesion molecules in oxLDL-elicited HUVECs. These observations indicate that berberine exerts its protective effect at various stages of atherosclerosis.

In conclusion, berberine disrupts the initiating process of atherosclerosis by attenuating adhesion of monocytes to endothelial cells and inhibiting expression of VCAM-1 and ICAM-1. These results provide new insights into this field of study that are likely to be used in the identification of novel drugs against atherosclerosis.

\section{Acknowledgements}

The current study was supported by grants from the National Natural Science Foundation of China (no. 81102837), the Traditional Chinese Medicine Administration of Zhejiang Province (no. 2010ZA085) and the Education Department of Zhejiang Province (no. Y201119884).

\section{References}

1. Price DT and Loscalzo J: Cellular adhesion molecules and atherogenesis. Am J Med 107: 85-97, 1999.

2. Cominacini L, Garbin U, Pasini AF, et al: Antioxidants inhibit the expression of intercellular cell adhesion molecule-1 and vascular cell adhesion molecule-1 induced by oxidized LDL on human umbilical vein endothelial cells. Free Radic Biol Med 22 117-127, 1997.

3. Jeng JR, Chang CH, Shieh SM and Chiu HC: Oxidized low-density lipoprotein enhances monocyte-endothelial cell binding against shear-stress-induced detachment. Biochim Biophys Acta 1178: 221-227, 1993.
4. Khan BV, Parthasarathy SS, Alexander RW and Medford RM: Modified low density lipoprotein and its constituents augment cytokine-activated vascular cell adhesion molecule-1 gene expression in human vascular endothelial cells. J Clin Invest 95: 1262-1270, 1995.

5. Takei A, Huang Y and Lopes-Virella MF: Expression of adhesion molecules by human endothelial cells exposed to oxidized low density lipoprotein. Influences of degree of oxidation and location of oxidized LDL. Atherosclerosis 154: 79-86, 2001.

6. Ross R: The pathogenesis of atherosclerosis: a perspective for the 1990s. Nature 362: 801-809, 1993.

7. Blankenberg S, Barbaux S and Tiret L: Adhesion molecules and atherosclerosis. Atherosclerosis 170: 191-203, 2003.

8. Takahashi M, Ikeda U, Masuyama J, Kitagawa S, Kasahara T, Shimpo M, Kano S and Shimada K: Monocyte-endothelial cell interaction induces expression of adhesion molecules on human umbilical cord endothelial cells. Cardiovasc Res 32: 422-429, 1996.

9. Hansson GK: Immune mechanisms in atherosclerosis. Arterioscler Thromb Vasc Biol 21: 1876-1890, 2001.

10. Ikram M: A review on the chemical and pharmacological aspects of genus Berberis. Planta Med 28: 353-358, 1975.

11. Stermitz FR, Lorenz P, Tawara JN, Zenewicz LA and Lewis K Synergy in a medicinal plant: antimicrobial action of berberine potentiated by 5'-methoxyhydnocarpin, a multidrug pump inhibitor. Proc Natl Acad Sci USA 97: 1433-1437, 2000.

12. Kong W, Wei J, Abidi P, et al: Berberine is a novel cholesterollowering drug working through a unique mechanism distinct from statins. Nat Med 10: 1344-1351, 2004

13. Yin J, Xing $\mathrm{H}$ and Ye J: Efficacy of berberine in patients with type 2 diabetes mellitus. Metabolism 57: 712-717, 2008.

14. Lee CH, Chen JC, Hsiang CY, Wu SL, Wu HC and Ho TY: Berberine suppresses inflammatory agents-induced interleukin-1beta and tumor necrosis factor-alpha productions via the inhibition of IkappaB degradation in human lung cells. Pharmacol Res 56: 193-201, 2007.

15. Huang Z, Wang L, Meng S, Wang Y, Chen $T$ and Wang C: Berberine reduces both MMP-9 and EMMPRIN expression through prevention of p38 pathway activation in PMA-induced macrophages. Int J Cardiol 146: 153-158, 2011.

16. Hsieh YS, Kuo WH, Lin TW, Chang HR, Lin TH, Chen PN and Chu SC: Protective effects of berberine against low-density lipoprotein (LDL) oxidation and oxidized LDL-induced cytotoxicity on endothelial cells. J Agric Food Chem 55: 10437-10445, 2007.

17. Pamukcu B, Lip GY, Devitt A, Griffiths H and Shantsila E: The role of monocytes in atherosclerotic coronary artery disease. Ann Med 42: 394-403, 2010.

18. Mitra S, Deshmukh A, Sachdeva R, Lu J and Mehta JL: Oxidized low-density lipoprotein and atherosclerosis implications in antioxidant therapy. Am J Med Sci 342: 135-142, 2011.

19. Mitra S, Goyal T and Mehta JL: Oxidized LDL, LOX-1 and atherosclerosis. Cardiovasc Drugs Ther 25: 419-429, 2011.

20. Fotis L, Agrogiannis G, Vlachos IS, et al: Intercellular adhesion molecule (ICAM)-1 and vascular cell adhesion molecule (VCAM)-1 at the early stages of atherosclerosis in a rat model. In Vivo 26: 243-250, 2012.

21. Chen H, Li D, Saldeen T and Mehta JL: Transforming growth factor-beta(1) modulates oxidatively modified LDL-induced expression of adhesion molecules: role of LOX-1. Circ Res 89: 1155-1160, 2001.

22. Li D, Chen H, Romeo F, Sawamura T, Saldeen T and Mehta JL: Statins modulate oxidized low-density lipoprotein-mediated adhesion molecule expression in human coronary artery endothelial cells: role of LOX-1. J Pharmacol Exp Ther 302: 601-605, 2002.

23. Steffens $\mathrm{S}$ and Mach F: Inflammation and atherosclerosis. Herz 29: 741-748, 2004.

24. Anis KV, Rajeshkumar NV and Kuttan R: Inhibition of chemical carcinogenesis by berberine in rats and mice. J Pharm Pharmacol 53: 763-768, 2001.

25. Yin J, Gao Z, Liu D, Liu Z and Ye J: Berberine improves glucose metabolism through induction of glycolysis. Am J Physiol Endocrinol Metab 294: E148-E156, 2008.

26. Chen FL, Yang ZH, Liu Y, et al: Berberine inhibits the expression of TNFalpha, MCP-1 and IL-6 in AcLDL-stimulated macrophages through PPARgamma pathway. Endocrine 33: 331-337, 2008.

27. Newby AC: Metalloproteinase expression in monocytes and macrophages and its relationship to atherosclerotic plaque instability. Arterioscler Thromb Vasc Biol 28: 2108-2114, 2008. 